\title{
Morphometric Definition of Alzheimer's Disease Stages by Means of The Tomography Dementia Rating Scale (TDR)
} Ivan V Maksimovich MD*

Department of Interventional Neuroangiology, Clinic of Cardiovascular Diseases named after Most Holy John Tobolsky, Moscow, Russia

\begin{abstract}
Background: The research is dedicated to developing an objective method for determining dementia severity in patients with different $A D$ stages. The method is based on morphometric analysis of specific atrophic changes in temporal lobes detected during cerebral CT and MRI and it allows differentiating these particular changes from those common for other cerebral neurodegenerative diseases.
\end{abstract}

Materials and Methods: 1105 patients aged 28 years to 81 years (mean age 75) were examined: 786 men (71.13\%), 319 women (28.61\%), 93 had different AD stages - Test Group, 1012 had other neurodegenerative diseases - Control Group.

Results: The scale of dementia stages during AD, Tomography Dementia Rating Scale (TDR), was developed, allowing to determine dementia severity with objective, morphometrically grounded data of atrophic changes in temporal lobes obtained during CT and MRI:

1. Preclinical AD stage-TDR-0: results from atrophic changes in temporal lobes with $4 \%$ to $8 \%$ tissue mass decrease and cognitive functions decline equal to 26 to 28 MMSE points.

2. Early $A D$ stage-TDR-1: mild dementia resulting from atrophic changes in temporal lobes with $9 \%$ to $18 \%$ tissue mass decrease, corresponds to CDR-1, is accompanied by cognitive functions decline equal to 20 to 25 MMSE points.

3. Middle AD stage-TDR-2: moderate dementia resulting from atrophic changes in temporal lobes with $19 \%$ to $32 \%$ tissue mass decrease, corresponds to CDR-2, cognitive functions decline is equal to 12 to 19 MMSE points.

4. Late AD stage-TDR-3: severe dementia resulting from atrophic changes in temporal lobes with $33 \%$ to $62 \%$ tissue mass decrease, corresponds to CDR-3, cognitive functions decline is equal to MMSE 7 to 11 points.

Control Group patients did not have any similar changes.

Conclusion: The proposed objective, morphometrically validated TDR scale allows to identify preclinical and clinical AD stages; it is easy to use and is complementary to the Clinical Dementia Rating scale. Besides, this scale makes it possible to differentiate AD from other neurodegenerative diseases.

Keywords: Alzheimer's disease; Dementia; Preclinical Alzheimer's disease; Stages of AD; Hippocampus; TDR; CDR; Atrophy of the temporal lobes of the brain

\section{Introduction}

$\mathrm{AD}$ is one of the leading neurodegenerative diseases. In recent years, there are over 36 million people suffering from this disease [1]. In the USA, AD has been diagnosed in 5 million people, $5 \%$ of whom acquire the disease at a fairly young age [2].

The ethology and pathogenesis of AD are not fully understood. The use of CT, MRI, PET, the introduction of biomarkers, and research on genetically modified mice have made it possible to achieve significant success in studying changes in the structure of temporal lobes and in the brain in general, in the distribution of beta and tau amyloid in them, but there are still many unresolved issues [3-13].

Preclinical AD stage is the most difficult to diagnose. This stage proceeds quite secretly; it begins with minor involutive changes in temporal cerebral lobes long before clinical manifestations of the disease; it does not have a clear clinical picture; it is difficult to differentiate it from other neurodegenerative diseases and to determine the severity of the lesion accurately [3,13-17].

Over the past few years, the most common clinical practice for determining the severity of dementia is the Clinical Dementia Rating scale (CDR) [18]. This method entices by its simplicity, but its disadvantages are the subjectivity of the assessment of visible disorders and its ineffectiveness in the diagnosis of the preclinical AD stage [17].

In the world clinical practice, there have been studies aimed at more objective determination of $\mathrm{AD}$ stages. Comparative characteristics of atrophic changes in cerebral temporal lobes were made based on analysis of MRI data and autopsy data [6]. There have been studies to determine the $\mathrm{AD}$ stages, which were based on MRI analysis of atrophic changes developing throughout the brain [19]. However, these studies did not allow a clear distinction of the stages of the disease. Consequently, there remains a need to develop some new, objective methods that enable the determination of the severity of dementia in any stage of $\mathrm{AD}$ which simultaneously allow the differentiation of $\mathrm{AD}$ from other neurodegenerative diseases.

*Corresponding author: Ivan V. Maksimovich, Department of Interventional Neuroangiology, Clinic of Cardiovascular Diseases named after Most Holy John Tobolsky, Moscow, Russia. Tel: +7(495)4390945; E-mail: carvasc@yandex.ru

Received June 29, 2017; Accepted July 31, 2017; Published August 05, 2017

Citation: Maksimovich IV (2017) Morphometric Definition of Alzheimer's Disease Stages by Means of The Tomography Dementia Rating Scale (TDR). Brain Disord Ther 6: 238. doi: 10.4172/2168-975X.1000238

Copyright: (c) 2017 Maksimovich IV. This is an open-access article distributed under the terms of the Creative Commons Attribution License, which permits unrestricted use, distribution, and reproduction in any medium, provided the original author and source are credited. 
The purpose of this research is to develop an objective method for determining the severity of dementia in patients with various $\mathrm{AD}$ stages, which is based on morphometric analysis of specific atrophic changes in temporal lobes detected during cerebral CT and MRI and to differentiate these changes from those occurring during other common neurodegenerative diseases.

Besides, the purpose of the research is to develop a scale of dementia severity in AD, the Tomography Dementia Rating scale (TDR), based on objective CT and MRI data.

\section{Materials and Methods}

All the studies accomplished during this research were carried out with the approval of the ethics committee, as well as with the consent of the examined patients and their relatives. 1105 patients aged 28 to 81 (mean age 75 years) were examined: $786(71.13 \%)$ men and $319(28.61 \%)$ women with neurodegenerative lesions of the brain of different genesis.

\section{Patient Examination}

\section{Examination plan}

1. Clinically, the severity of dementia was assessed in accordance with "The Clinical Dementia Rating scale" (CDR) [18].

2. Cognitive functions were determined using the "Mini-Mental State Examination" (MMSE) method [19].

3. Coagulatory, biochemical and general clinical data were obtained through laboratory methods of research [13].

4. Brain scintigraphy (SG) was performed according to the classical method in a dynamic and static mode on a gamma camera of "Ohio Nuclear" (USA) using TC 99M per technetate 555 [17].

5. Rheoencephalography (REG) was performed in standard modes identifying disorders in pulse blood filling in the cerebral hemispheres, on the apparatus "Reospectr-8" ("Neurosoft", Russia) [13].

6. CT and MRI of the brain were performed on the tomographs "Somatom" (Siemens), "Hi Speed" (GE), "Tomoscan" (Philips), and "Apetro Eterna" (Hitachi). The ATAA (Advance Tomo Area Analysis) method [20] was used to perform the examination, allowing to determine the percentage of the decrease in tissue mass of temporal lobes in comparison with their normal volume, thus showing the severity of atrophic changes [21,22].

The gist of the method is the following: during CT or MRI, the boundary of the first scan goes along the orbital-myotonic line. Earlier, the apparatus used allowed scans of $1 \mathrm{~mm}$ or $2 \mathrm{~mm}$ thick, with an interval of $1 \mathrm{~mm}$ or $2 \mathrm{~mm}$ [21]. The apparatus of a later making produced scans of $1 \mathrm{~mm}$ thick, without any interval between scans. The bones serve as the boundaries of each of the middle cranial fossa. Using the computer program Advance Tomo Area Analysis (ATAA) [17], the area of the middle cranial fossa and the size of the brain substance of each temporal lobe on each scan are measured [21]. Further, the area of the lower horn of the lateral ventricle and the area of the fissures are subtracted, after which the obtained data are compared with the area of the corresponding cranial fossa on the same scan. The scans are summed. The automatically obtained measures are recalculated by the thickness of each scan and each interval between the scans (if any), and then the volume and mass of the cerebral tissue in the area under investigation are determined. The ratio of these measures allows us to determine the state of the brain tissue, both normal and adjusted for the total share of intracranial volumes, to consider them in the development of atrophic processes. As a result, the normal volume of the cerebral tissue for the corresponding age group is determined, as well as the percentage reduction in the tissue mass of the cerebral temporal lobes and the hippocampus in $\mathrm{AD}$. By the ratio of the obtained measures, the percentage of the decrease in the volume of the cerebral tissue in the investigated area is determined. Since the patients examined belonged to different age groups, the study took into account age-related changes in brain tissue [22]. Next, comparison is made between the percentage reduction in the temporal lobe volume and the clinical picture of dementia on the CDR scale, resulting in an objective, morphometrically valid assessment of the dementia stage in $\mathrm{AD}$ [23].

\section{Test group}

$93(8.42 \%)$ patients aged 34 years to 79 years (mean age 75 years): 32 (34.41\%) men, 61 (65.59\%) women with different stages of AD:

$1.10(10.75 \%)$ had pre-clinical AD stage.

2. 26 (27.96\%) had mild dementia equal to CDR-1.

3. $40(43.01 \%)$ had moderate dementia equal to CDR-2.

4. $17(18.28 \%)$ had severe dementia CDR.

\section{Control group}

$1012(91.58 \%)$ people aged 28 years to 81 years (average age 73 years): $588(58.10 \%)$ men, $424(41.90 \%)$ women suffering from neurodegenerative diseases comparable in severity worsened by cerebral involuntary changes, dementia and cognitive disorders, but not AD. Etiologically, the patients were divided in the following way:

$1.27(2.67 \%)$ people showed the initial signs of chronic cerebrovascular insufficiency of atherosclerotic genesis unaccompanied by severe dementia and cognitive impairment.

$2.577(57.02 \%)$ patients showed pronounced signs of chronic cerebrovascular insufficiency of atherosclerotic genesis. Dementia at CDR1 level was detected in $262(45.41 \%)$ patients, at CDR-2 level in 315 (54.59\%) patients, which was accompanied by a decrease in cognitive functions to 18 to 25 MMSE points.

$3.342(33.80 \%)$ patients had a severe form of chronic cerebrovascular insufficiency. Dementia at CDR-2 level was detected in 253 (73.98\%) patients, at the level of CDR-3 in 89 (8.79\%) patients, which was accompanied by a decrease in cognitive functions to 11 to 19 MMSE points.

4. 23 (2.27\%) patients had signs of Binswanger's disease. Dementia at CDR-1 level was detected in $16(69.57 \%)$ patients, at CDR-2 level in $7(30.43 \%)$ patients, which was accompanied by a decrease in cognitive functions to 12 to 25 MMSE points.

5. 34 (3.36\%) patients had signs of vascular Parkinsonism. Dementia at CDR-1 level was detected in 18 (52.94\%) patients, at CDR2 level in $16(47.06 \%)$ patients, which was accompanied by a decrease in cognitive functions to 18 to 25 MMSE points.

6. $9(0.89 \%)$ patients demonstrated signs of Parkinson's disease. Dementia at CDR-1 level was detected in 5 (55.56\%) patients, at CDR-2 level in $4(44.44 \%)$ patients, which was accompanied by a decrease in cognitive functions to 16 to 25 MMSE points.

\section{Results}

\section{Test group}

CT and MRI showed specific changes in the brain in all cases of patients with $\mathrm{AD}$, which can be divided into 2 groups: 


\begin{tabular}{|c|c|c|c|}
\hline Cerebral changes & $\begin{array}{c}\text { Test } \\
\text { group } \\
\mathrm{N}-93\end{array}$ & $\begin{array}{c}\text { Control } \\
\text { group } \\
\mathrm{N}-1012\end{array}$ & $\begin{array}{l}P \text { (chi- } \\
\text { square) }\end{array}$ \\
\hline \multicolumn{4}{|c|}{ Changes in temporal areas } \\
\hline $\begin{array}{l}\text { Local involutive changes of the brain cortex in } \\
\text { temporal areas }\end{array}$ & 93 & 0 & \multirow{5}{*}{$p<0.01$} \\
\hline $\begin{array}{l}\text { Local atrophy of temporal lobes with } 4 \% \text { to } 8 \% \\
\text { decrease in tissue mass (TDR-0) }\end{array}$ & 10 & 0 & \\
\hline $\begin{array}{l}\text { Local atrophy of temporal lobes with } 9 \% \text { to } \\
18 \% \text { decrease in tissue mass (TDR- } 1 \text { ) }\end{array}$ & 26 & 0 & \\
\hline $\begin{array}{l}\text { Local atrophy of temporal lobes with } 19 \% \text { to } \\
32 \% \text { decrease in tissue mass (TDR-2) }\end{array}$ & 40 & 0 & \\
\hline $\begin{array}{l}\text { Local atrophy of temporal lobes with } 33 \% \text { to } \\
62 \% \text { decrease in tissue mass (TDR-3) }\end{array}$ & 17 & 0 & \\
\hline \multicolumn{4}{|c|}{ General cerebral changes } \\
\hline $\begin{array}{l}\text { Multiple calcium salts deposits in intracranial } \\
\text { vessels }\end{array}$ & 0 & 998 & \multirow{4}{*}{$p<0.01$} \\
\hline $\begin{array}{l}\text { Single and multiple post-ischemic microcysts } \\
\text { (more than } 5 \mathrm{~mm} \text { ) }\end{array}$ & 0 & 132 & \\
\hline Post-ischemic microcysts of less than $5 \mathrm{~mm}$ & 0 & 148 & \\
\hline $\begin{array}{l}\text { General neurodegenerative changes in the } \\
\text { cortex of the brain }\end{array}$ & 48 & 876 & \\
\hline Sylvian fissures widening signs & 93 & 974 & $\begin{array}{c}\text { Not } \\
\text { significant }\end{array}$ \\
\hline Leucoaraiosis signs & 0 & 178 & \multirow{2}{*}{$p<0.01$} \\
\hline Unocclusive hydrocephaly signs & 57 & 453 & \\
\hline
\end{tabular}

To define the differences in the evidence (between Test Group and Control Group), we conducted an analysis of contingency tables with Chi-square criterion. All the signs except for 'Sylvian fissures widening signs' revealed significant diffirences $(p<0.01)$. The statistical analysis was accomplished with the help of Statsoft Statistica 10 program.

Table 1: CT and MRI data in test and control group patients.

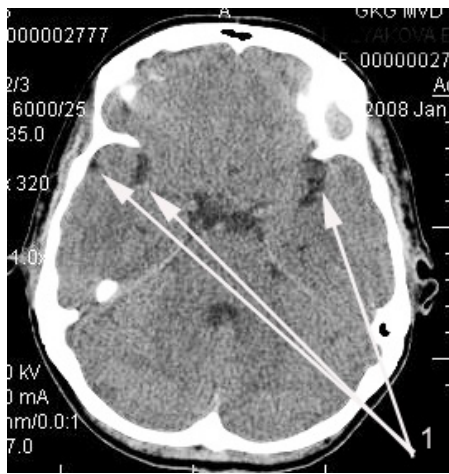

Figure 1: Patient $\mathrm{H}$, female, 40-year-old: $\mathrm{CT}$ of the brain. Pre-clinical stage TDR-0 (tissue mass decrease: right temporal lobe- $6 \%$, left temporal lobe- $8 \%$ ) 1) Marginal atrophy.

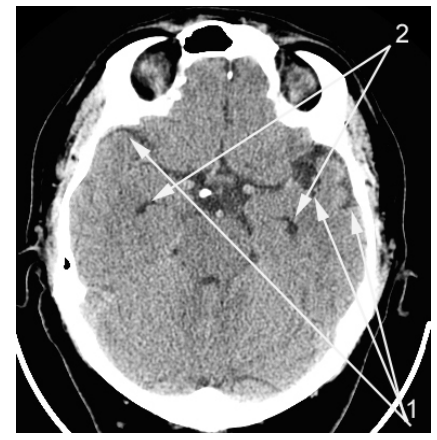

Figure 2: Patient S, female, 52-year-old: CT of the brain. Early stage TDR1 (tissue mass decrease: right temporal lobe- $9 \%$, left temporal lobe-14\%) 1) Marginal atrophy 2) Atrophy in the form of a cavity.

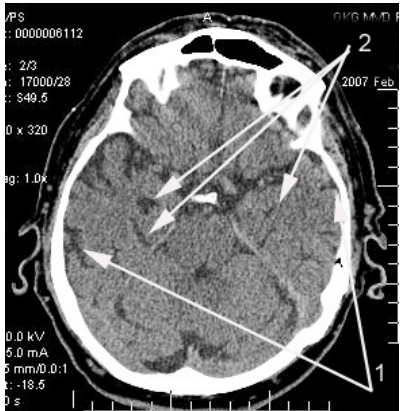

Figure 3: Patient $\mathrm{K}$, male, 58-year-old: CT of the brain. Middle stage TDR-2 (tissue mass decrease: right temporal lobe-27\%, left temporal lobe-20\%) 1) Marginal atrophy 2) Atrophy in the form of a cavity.

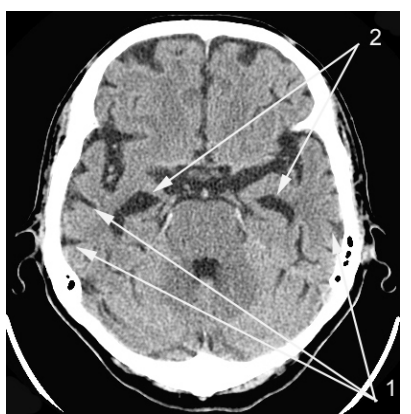

Figure 4: Patient $\mathrm{P}$, female, 72-year-old: CT of the brain. Late stage TDR-3 (tissue mass decrease: right temporal lobe-42\%, left temporal lobe-38\%). 1) Marginal atrophy 2) Atrophy in the form of a cavity.

1. Atrophic changes in temporal lobes, which, depending on the stage of the disease, lead to tissue mass decrease from 4 to $62 \%$ (Table 1 ). In the early stages of $\mathrm{AD}$, these changes are manifested by peripheral, marginal atrophy (Figure 1). In the later stages of $\mathrm{AD}$, atrophic changes lead to the formation of single and multiple cavities in the tissue of temporal lobes, ranging from 1 to $20 \%$ of the total volume of the lobe (Figures 2, 3, 4);

\section{General cerebral changes (Table 1).}

Based on the results of the study, a scale of the stages of dementia in the development of $\mathrm{AD}$ has been worked out, "The Tomography Dementia Rating Scale" (TDR). The use of this scale allows determining the severity of dementia by objective, morphometrically grounded data of atrophic changes in cerebral temporal lobes obtained during CT and MRI:

1. Preclinical AD stage-TDR-0: results from atrophic changes in temporal lobes with $4 \%$ to $8 \%$ tissue mass decrease (Figure 1 ) and cognitive functions decline equal to 26 to 28 MMSE points.

2. Early AD stage-TDR-1: mild dementia resulting from atrophic changes in temporal lobes with $9 \%$ to $18 \%$ tissue mass decrease, corresponds to CDR-1 (Figure 2), is accompanied by cognitive functions decline equal to 20 to 25 MMSE points.

3. Middle AD stage-TDR-2: moderate dementia resulting from atrophic changes in temporal lobes with $19 \%$ to $32 \%$ tissue mass decrease (Figure 3), corresponds to CDR-2, cognitive functions decline is equal to 12 to 19 MMSE points.

4. Late AD stage-TDR-3: severe dementia resulting from atrophic changes in temporal lobes with $33 \%$ to $62 \%$ tissue mass decrease, corresponds to CDR-3 (Figure 4), cognitive functions decline is equal to MMSE 7 to 11 points. 


\section{Control group}

CT and MRI showed that the patients had multiple morphological changes in the brain related to general cerebral changes. An analysis of these changes is presented in Table 1.

Specific atrophic changes in the tissue of the temporal lobes of the brain characteristic for the development of AD were not detected.

\section{Discussion}

The data obtained during the research show that the patients of the test and control groups have distinct differences of the developed morphological and structural disorders in the brain.

Specific structural changes of atrophic nature develop in the temporal cerebral areas in the patients of the test group in all AD stages. These changes allow to differentiate the stages of the disease. These changes are manifested in a decrease in the mass of the temporal lobes and hippocampus tissue, which develops along with the progression of the stage of the disease.

The degree of manifestation of these changes is directly dependent on the stage of $\mathrm{AD}$, the severity of dementia and the severity of cognitive disorders $[13,17,21]$. Each stage of AD corresponds to a certain decrease in the tissue mass of temporal lobes, expressed as a percentage of the norm $[22,23]$.

These specific atrophic changes of temporal lobes are not found in control group patients who have other neurodegenerative brain lesions accompanied by dementia and cognitive disorders. Involuntary cerebral changes in control group patients are of a general cerebral character and in the overwhelming majority of cases are manifested by general hypotrophy and atrophy of all cerebral structures.

The presented data are confirmed by studies of other authors who showed the presence of atrophic changes in temporal lobes in patients suffering from $\mathrm{AD}[3,12,16,19]$ and the absence of similar atrophic changes in patients suffering from neurodegenerative lesions of another etiology such as dementia with Lewi bodies and vascular cognitive lesions [6].

\section{Conclusion}

The proposed objective, morphometrically validated TDR scale is accurate enough; it allows to identify preclinical and clinical AD stages, it is easy to use, and is complementary to the Clinical Dementia Rating scale, which significantly facilitates the diagnosis of the stages of the disease. Besides, this scale makes it possible to differentiate AD from other neurodegenerative diseases.

\section{Conflict of Interest}

The authors declare no conflict of interest.

\section{References}

1. Alzheimer's Association (2017) Alzheimer's disease facts and figures. J Alzheimer's \& Dementia 13: 325-373.

2. Grammas P, Martinez J, Sanchez A, Yin X, Riley J, et al. (2014) A new paradigm for the treatment of Alzheimer's disease: Targeting vascular activation. J Alzheimers Dis 40: 619-630.

3. Mormino EC, Papp KV, Rentz DM, Donohue MC, Amariglio R, et al. (2017) Early and late change on the preclinical Alzheimer's cognitive composite in clinically normal older individuals with elevated amyloid- $\beta$. J Alzheimer's \& Dementia

4. Jack CR, Petersen RC, Xu YC, O'Brien PC, Smith GE, et al. (1999) Prediction of $A D$ with MRI-based hippocampal volume in mild cognitive impairment. Neurology 5: 1397-1403

5. Waldemar G, Dubois B, Emre M, Georges J, McKeith IG, et al. (2007) Recommendations for the diagnosis and management of Alzheimer's disease and other disorders associated with dementia: EFNS guideline. Eur J Neurol 14: e1-e26.

6. Burton EJ, Barber R, Mukaetova-Ladinska EB, Robson J, Perry RH, et al. (2009) Medial temporal lobe atrophy on MRI differentiates Alzheimer's disease from dementia with Lewy bodies and vascular cognitive impairment: A prospective study with pathological verification of diagnosis. Brain 132: 195-203.

7. Trojanowski JQ, Vandeerstichele $\mathrm{H}$, Korecka M, Clark $\mathrm{CM}$, Perry $\mathrm{RH}$, et al. (2010) Update on the biomarker core of the Alzheimer's disease neuroimaging initiative subjects. Alzheimers Dement 6: 230-238.

8. Meyer PT, Hellwig S, Amtage F, Rottenburger C, Sahm U, et al. (2011) Dualbiomarker imaging of regional cerebral amyloid load and neuronal activity in dementia with PET and 11C-labeled Pittsburgh compound. J Nucl Med 52 393-400.

9. Adriaase A, Sanz-Arigita E, Binnewijzend M, Tolboom N, Wink AW, et al. (2011) Molecular markers of Alzheimer's disease pathology and their relationship with default mode network integrity. Alzheimers Dement 7: S2-S3.

10. Weiner WW, Veitch DP, Aisen PS, Beckett LA, Nigel JC, et al. (2015) 2014 Update of the Alzheimer's disease neuroimaging initiative: A review of papers published since its inception. Alzheimers Dement 11e1-11e120.

11. Jun G, Naj FC, Beecham GM, Wang LS, Buros J, et al. (2010) Meta-analysis confirms CR1, CLU, and PICALM as Alzheimer disease risk loci and reveals interactions with APOE genotypesю. Arch Neurol 67: 1473-1484.

12. Shen L, Fipri HA, Saykin AJ, West JD (2009) Parametric surface modeling and registration for comparison of manual and automated segmentation of the hippocampus. Hippocampus 19: 588-595.

13. Maksimovich IV (2008) Radiodiagnostics of Alzheimer's disease. Diagn Interv Radiol 2: 27-38.

14. Mayeux R, Reitz C, Brickman AM, Haan MN, Manly JJ, et al. (2011) Operationalizing diagnostic criteria for Alzheimer's disease and other agerelated cognitive impairment Part 1. Alzheimers Dement 7: 15-34.

15. Seashadri S, Beaser A, Au R, Volf PA, Evans DA, et. al. (2011) Operationalizing diagnostic criteria for Alzheimer's disease and other age-related cognitive impairment-Part 2. Alzheimers Dement 7: 35-52.

16. Annear MJ, Eccleston CE, Mclnerney FJ, Elliott KE, Toye CM, et al. (2016) A new standard in dementia knowledge measurement: Comparative validation of the dementia knowledge assessment scale and the Alzheimer's disease knowledge scale. J Am Geriatr Soc 64: 1329-1334.

17. Maksimovich IV (2012) Certain new aspects of etiology and pathogenesis of Alzheimer's disease. Adv Alzheimer Dis 1: 68-76.

18. Morris JC (1993) The clinical dementia rating (CDR): Current version and scoring rule. Neurology 43: 2412-2414.

19. Chiang GC, Insel PHS, Tosun D, Schuff N, Sacrey DT, et al. (2011) Identifying cognitively healthy elderly individuals with subsequent memory decline by using automated MR temporoparietal volumes. Radiology 259: 844-51.

20. Folstein MF, Folstein SE, McHugh PR (1975) Mini-mental state. A practical method for grading the cognitive state of patients for the clinician. J Psychiatr Res 12: 189-198

21. Maksimovich IV, Gotman LN, Masyuk SM. (2006) Method of determining dimensions of temporal brain lobes in patients suffering from Alzheimer's disease. Russian Patent No 2306102.

22. Maksimovich IV, Gotman LN (2006) Method of complex radiation diagnostics at preclinical and clinical stages of Alzheimer's disease. Russian Patent No. 2315559.

23. Maksimovich IV (2012) The tomography dementia rating scale (TDR)- The rating scale of Alzheimer's disease stages. Health 4: 712-719. 